\title{
EL LEXICO DE LA LENGUA ANDAQUí
}

\author{
Gabriela Coronas Urzúa
}

\begin{abstract}
This articles presents an alphabetical arrangement of the entire lexicon of Andaqui (an extinct indigenous Colombian language) contained in two documents sent to Spain during colonial times. These data constitute the only sources avaliable to analyse this languaje. The dictionary includes two parts: the Andaquí-Spanish vocabulary and the Spanish-Andaquí vocabulary.
\end{abstract}

\section{Introducción}

La lengua andaquí es una de las muchas lenguas indígenas actualmente extintas que se hablaban en América a la llegada de los españoles.

En las últimas décadas del siglo XVIII y la primera mitad del siglo XIX, cuando fueron recogidos los datos que sirven de base a este estudio, las tribus hablantes de la lengua andaquí (Aguanunga y Charaguaye), habitaban la región de la selva amazónica colombiana adyacente al Alto Caquetá, lo que hoy constituye los Departamentos del Putumayo y del Caquetá. Así se desprende del estudio hecho por Friede (1953: 71-79), basado en documentos que relatan la visita del Gobernador Don Diego de Ospina a la región por los años 1628 y 1629 , en el que aporta numerosas razones para pensar que otras etnias a las que también se dio el nombre de andaquíes y que habitaban las tierras adyacentes al Alto Magdalena no hablaban la misma lengua. (Coronas: 1994)

Los dos documentos estudiados para la realización de este trabajo constituyen las únicas fuentes disponibles para el análisis de la lengua; son dos listas de vocabulario andaquí recogido por misioneros españoles. El primer documento, anónimo, fue enviado a España en 1787 por don José Celestino Mutis, cuyos datos, afirma Friede (1953: 69) "le fueron suministrados por los misioneros franciscanos que en aquel tiempo tenían a su cargo la conversión y 'reducción' de los Andakí del Caquetá" (Anónimo: 1928); el segundo fue recogido y publicado por el Padre Manuel María Albis, quien viajó al Caquetá en 1854 (Albis 1855: 53-72). De este último, la versión consultada está traducida al inglés. 
El estudio realizado por Paul Rivet del documento del Padre Albis anteriormente citado, lo condujo a proponer la clasificación del andaquí en el grupo aruak-chibcha (Rivet: 1924: 106). Esta relación del andaquí con otras lenguas incluidas en el filo macrochibcha ha sido mantenido hasta el presente; sin embargo, Constenla (1981: 12) afirma que "...la subclasificación con el muisca y las lenguas de la Sierra Nevada de Santa Marta es completamente errónea". La tesis de la relación del andaquí con las lenguas chibchas se basa en supuestas semejanzas determinadas mediante el método de inspección; pero hasta el momento no se ha aplicado para su estudio el método comparativo.

$\mathrm{El}$ análisis del vocabulario contenido en dichos documentos permitió, en una primera etapa, el establecimiento del sistema fonológico del andaquí (Coronas: 1994), mediante la aplicación del método que Adolfo Constenla ha propuesto denominar "restitución" (comunicación personal ). A partir del establecimiento del sistema fonológico fue posible realizar la transcripción fonemática de todo el vocabulario contenido en los dos documentos citados. En este trabajo se presenta, integrado en un solo documento, debidamente ordenado de acuerdo con los métodos de la lexicografía moderna, como lo expone Haensch (1982: 395-534), y dividido en dos partes que corresponden, respectivamente, al léxico andaquí-español y al léxico español-andaquí. En el primero de ellos, los morfemas radicales se han numerado y ordenado alfabéticamente según la transcripción fonemática empleada. Cada artículo hace mención de la fuente y de las transcripciones en que el material aparece registrado.

En la parte correspondiente al léxico español-andaquí, éste se presenta ordenado alfabéticamente según la ortografía castellana. Junto a la transcripción fonemática del andaquí, se indica el número correspondiente a la entrada de cada artículo en la versión del léxico andaquí-español.

En la transcripción fonemática se empleó el alfabeto de la Asociación Fonética Internacional (1949).

\subsection{Abreviaturas}

En el léxico correspondiente a la parte andaquí-español se identifica el vocabulario contenido en el documento del Presbítero Manuel María Albis con la abeviatura: [A].

\subsection{Sobre la grafía en la transcripción}

En la transcripción al castellano se ha empleado la ortografía actual, no la de la fuente colonial cuya conservación carece de sentido en este trabajo, pues trata sobre el andaquí y no sobre el castellano de la época. 


\section{Léxico Andaquí- Español}

A

1. aa haa

2. ahi

3. ahu

4. ahubinaka

5. ahuma-

6. aisimi-si

7. akai

8. akusinipii

9. akwajuhu

10. ani

11. asahui

12. atat $\mathrm{i}$

13. ãta-

14. ãtaku

15. ãti-

16. ãtuasu

17. ãsiha nípikwa 'sí ' (ahá 'sí quiero'; jahá ' sî')

'brazo de río ' (axi)

'quebrada' (axó)

'muelas; molares' ([A] ajivenaca)

'pereza' (juajumacaque 'perezoso’; neajumachi? ‘tienes pereza?')

'azul' (aiszimi-zi)

'limón ' ([A] acai )

'bellaco' (acozinipihi)

'medias o botas' (aguayoxo)

'haber' (bacoxe hane? ‘hay chicha?’; noszuejane? ‘ hay pescado?')

'Dios' ( [A] asajué)

'por la mañana' (atachi)

'alzar' (anta-zá ‘alza!')

'presto, rápido, de prisa' (dacoza, andagu, antagoni)

'gritar' (ante-zá 'grita')

'plátano' (anduazo, duazo, anduozo)

'puta o ramera' (hanszexa nimpigua)

B

18. bahii

19. bahũpi-

20. bahuat $\mathrm{i}$

21. bahuaka bajaka

22. bakuhi

23. bakwĩhi

24. bakwi

25. bakuja

26. bakusa

27. banũkưtu

28. basaki

29. basuhi

30. batana

31. batuja

32. batunahi 'guacamaya ' ([A] bafe)

'desatar ' (baxumpe-zá 'desata')

'estar cansado' (ringa bajo hachi 'yo estoy cansado')

'flojo' (bayaga quihi, bajoaga quihi 'eres flojo')

'chicha' (bacoxe)

'miel de abejas' (bacuhenxe)

'miel' (bacuhi)

'abejas' (bacoya)

'mazato' (bacoza, bacuchi)

'llanto' (banûcuchú)

'culebra' ([A] basague)

'aguacero; chaparrón' ([A]basuji )

'batea' ([A] batana)

'carbón' (batoya)

'plato' ([A] batonafi ; batonaffi ) 
33. batui

34. bi-

35. bianaha

36. bihi-

37. bikai

38. bikwara

39. binitu

40. binihi

41. birajui

42. bisasi-

43. bisatui

44. bisi-

45. bisika

46. bitunahi

47. bity i-

48. bî́tikwai $\sim$ mãtikwai

49. buhưsi-

50. buhukaa

51. buhusaha-

52. buitre-ni

53. bujaţanai

54. bukwaru

55. bukwakai

56. bưta- 'brasa o fuego' (bathohé)

'cargar' (bi-zá 'carga')

'frío' (benaxa, beanaxá)

'correr' (bijiza 'corre')

'dorada' ( [A] vegaé; bicahi)

'mucho' (biguara)

'fierro' (binintó)

'machete' ([A] benije ; binixi)

'conejo' (verayuhé, verayue-ja 'el conejo') [CD]

‘cuánto' (bizazini? ‘¿qué tanto?’)

'pulgas' (biszatuhi)

'cuántos' (biszibujahá nifihichini? ‘¿cuántos días hay de camino?) bisikwini biszicuhini? ‘¿cuántos son?’)

'soy hombre' (biszica)

'macana' (bitonaxi)

'medir' (bichi-zá 'mide')

'murciélago' (bintiguay; [A] mandeguae)

'levantarse' (bujunszizá 'levántate')

'hacha' ([A] bojoca ; bujucahá, bujicá)

'bajar' (buju; buju sajaza 'baja')

'buitre' (buytre-ni)

'dátil' ([A] buyachanae')

'barba' (buquaro)

'perdiz pequeña' ([A] buguagai)

'dormir ' (bondazá 'duerme'; qua-buntahá 'anda, duerme')

H

57. haa

58. haakũ-

59. hahana

60. hajakasu

61. hakựi

62. hakwasi-

63. hana jana

64. harakwãi

65. hasijunitrini

66. hãjūi

67. hi- 'a ' [acusativo o dativo] (xa; rica-xá 'a ti' ; ringa-xa 'de mí')

‘curar’ (jahá cuncahá? ‘¿sabéis curar?’)

'llano' (jaxana)

'plátano artón' (jayacazú)

'harto' (jacóche)

'descansar' (jagua zibahá 'descansaremos')

'llanos' (jana)

'gallina' (jaraguahé, araunhé; taranguehé; [A]

saraguañae)

'espaldas' (fasziyunichini)

'mate grande' (jañuhe)

'caminar' (jxi-zá 'camina'; jeaszua 'caminemos') 
68. hirahity

69. hisaha-

70. hiríhi-

71. hĩ̃̃su-

72. hihi

73. hihia

74. hihinatu

75. hihii-

76. hihui

77. hii-

78. hii

79. hitga-

80. hijuty i

81. hijutribi

82. hiku

83. hikwi

84. hikujãha

85. hikwasi

86. hinahutr $\mathrm{i}$

87. hinai

88. hisaraa

89. hisani

90. hisasa

91. hisi

92. hisikakwani

93. hisuĩã

94. hiti-si

95. hitii

96. hitinahu

97. hiusi

98. hinasa

99. hísikai

100. hî́sisi

101. hîsu-

102. hĩtikai 'hambre' ( firajichi ; firajichihi, firaxixhi; firajigui chaza 'tengo hambre' ; firafichi-za 'que tengo hambre'; firajigui chaza 'en teniendo hambre')

¿¿para qué?' (fizajaquin, yzajazini, bizejazini, huizana caraxini, ¿¿para qué llevas?)

'amarrar' (firinxe-zá 'amarra!') 'amar, querer' ([A] fianso-me 'yo te amo';ca-fianzu-hé ‘queréis?'; ra-fianza-nca? ‘ ¿por qué no quieres?’)

'agua' ([A] jiji; jixe, jexê)

'quien sabe' (hijya, jijyâ)

'hígado' ([A]jifinató)

'llamar' (fifihé 'te llama'; fifi-zá 'llama'; tifi-zá 'llama')

'raya ; pejerraya ' ([A] jijoe; jejohé)

'ir' (ji-bi ‘me voy'; jihi-zá 'anda!'; ca-xihé, ‘fuiseis?;

jea-zi 'sí fui')

'demonio ' ([A] gii; jihi)

'rozar' (ficha-rá 'a rozar; ficha-nará 'a la chagra ; fichahé

'está rozando ')

'chirides' ([A] fiyuchi)

'periquito' (fiyuchibi)

'iglesia' (jico-ra 'a la iglesia')

'cerca' (jicue)

'viento' ([A] jiconoja; ficoñaxá)

'sal' (jiguazi)

'sed' (ninga finajuche 'yo tengo sed')

'negro' ([A] jinae; jinahé )

'jagua' [árbol] (jiszarahá)

'porque' (hizani)

'negro, negra' (fiszas-za, fiszas-zá)

'candela '([A] jifi; xifi; xizi, fizi 'leña ')

'porque' (fizicaguani, hizani)

'estrella' ([A] fisoñá; fiszohé)

'colorado' (fiti-zi)

'pauji' (fitihi)

'rastrojo' (jitinaxu)

'perder' (kajiuszi? ‘¿perdiste?’)

'por la tarde' (jináza)

'cojo' (finszicahe)

'sucio' (finszizi)

'limpiar' (finszuzá 'limpia')

'tusa de maíz' (finticahé) 
103. hĩtu-

104. hĩtu-

105. hĩ̃a

106. hu-

107. hu-rapai

108. hũkira

109. hurahiha

110. hurukwakai

111. hãti

112. huana-hi

113. huanisi

114. huasi-

115. huatuha

116. hubi-

117. huirahu

118. hujakuu

119. hukwats iţ $\mathrm{i}$

120. hukwa saţua

121. hupinai

122. husaisa

123. husihii

124. husu

125. husuanipii

126. huũtaa

127. hưvikwa

128. hursunai

129. hưțika 'cortar' (fintozá 'corta')

'buscar' (fintura 'a buscar')

'avispas' (jiñâ)

'sembrar' (ju-zá 'siembra')

'lagarto, caimán' ([A] rapae; jorapahi)

'arriba' (junquerá, chitachini)

'caliente' (juraji-xa)

'perdiz' (jruguacahé)

'mucho' (juantizica 'harto hay')

'hombro' (johana; [A] juanafe)

'largo' (juanizi)

'lavar' (juaszi-za 'lava')

'derecho' (juatuxa)

'calentarse' (jubi-zá 'caliéntate')

'escoba' (fueraxú)

'poco' (raju yagohó 'poca gente hay',

zeanjeagu bizin cahá 'poco tengo')

'rascadera' (jogua chichi)

'diez días' (juguha fsachua)

'moscos' (jupenahé)

'calzones; pantalones' (josahesza; [A] josaesa )

'salir ' (juzijihi 'sale [el sol]')

'flauta' ([A] joso )

'gordo' (jushuanipihi)

'desnudo' (juhuntahá)

'anzuelo' ([A] ochegua; fuchigua)

'tábano' (juchunahé)

'árbol' (junchiga)

130. ihu

131. iisa

132. ikuhu

133. iraharu

134. isahura

135. isikara

136. if uisi

137. ĩbiina

'hamaca ' ([A] iju )

'por qué' (yhiza, hihiza; hihiza nazan canquinihi ‘por qué no avisaste?’)

‘molde para hacer perdigones '([A] icojó)

'acero' (yrajaró)

'mediodía' ( yzajora hihi 'ya es medio día')

'oro' (yszicará)

'qué' (ychuchi, ypchizé, ychuyzi, yffize,

ychuizi,ychuhizi, ychuize)

'plata ' ([A] imbina; bihina ) 
138. îsi

139. îtai

140. îf jua

141. ĩkihi

142. îkisi

143. sats $\mathrm{i}$

144. jaa

145. jaasa

146. jasi-

147. jasimi

148. jataa

149. jãũkaki

150. jisui

151. juhua-

152. juhui

153. juhi

154. ju

155. karupikwa

156. kaakii

157. kabii

158. kahi-

159. kaihi

160. kaţaa

161. katsika

162. kaţ ikai

163. kats inits $\mathrm{i}$

164. katf inikwa

165. kajãa

166. kaka

167. kaka

168. kaka- 'ir ' ([A]ince; ynszi, 'vámonos ; ynszi-za 'vamos')

'buitre' ([A] indai )

'mentira' (ynchua, inchua)

'nariz' (ynguifi; [A] quifi )

'carne' (ynguize)

'doler' (zinszache 'me duele', yazi mintzachi

'mucho me duele')

'río' ( yahara; yaha-ra 'al río')

'sî' (yahaza)

'malo', 'feo' ([A] yaseco; yazicu ; yazin kaqui 'eres fea')

'mucho' (yazi mi-)

'playa' (yatahá)

'grande' (jañucaque, janûca, janûgaqu; janûzira guanca

'no te pido harto')

'cargar' [el morral] ([A] yisoe)

'cargar ' [palos] (yojoa-za 'carga [este palo]')

'culebra' (yojohé)

'charco' (yoxé)

'venir' (ni-yu-hé? 'viene' ; ra-yo-ahi 'no viene'ca-yu-bi ‘¿vienes?'; ni-yo- aya 'ahí vienen')

$\mathbf{K}$

'tu mujer' (karupegua)

'saber' (aqueé, -caguehé, -cahá, cague ‘'sabéis?')

'loro' (cabihi; cabiyara 'los loros')

'lavar' (kaje-zá 'lava')

'mazamorra' (cahixi)

'madre' (cachaháxa 'a tu madre')

'caña brava' (cacheca)

'bagre pintadillo' ([A] cachicae)

'nieto' (cachineche)

'nieta' (cachinigua)

'gente; río' ([A] cañaa )

'tirar' (caca-za , kaca-zá 'tira o arroja')

'batata' (kagá)

'pescar' (kacará 'a pescar') 
169. kakajuhu

170. kakii

171. kamii-ra

172. kamini

173. kamija

174. kana-ra

175. kanaha

176. kanahisiha

177. kanu-

179. kasinai

180. katukwai

181. kãhuru

182. kãt ii

183. kãt ii rakwai

184. kãţibiha

185. kãkrihu

186. kãkihu

187. kãsu-

188. kãtihựi

189. kãtikaţ i

190. kia

191. kiaha

192. kihara

193. kii

194. kii

195. kiki

196. kikwa

197. kinaha

198. kinahi

199. kisiku

200. ku-

202. kubiha

203. kuhi

204. kuhuhi

205. kuku

206. kukwa

207. kukwi

208. kuma-

209. kupii 'cacao' ([A] lacayojó; cacahixo)

'sol' ([A] caqui; kaquihi )

'adentro' [locativo] (camihi-ra)

'alegre' (neamakamimi 'estoy alegre'nanqua kamimi?

¿'estás contento?)

'niguas' (camiya)

'abajo' (cana-ra)

'torcido' (canâxa)

'ayer ' (canajizexa, canajisexa)

'amolar' (cano-zá 'amuela el hacha')178. kapanai

'guacamaya' ([A] capanae)

'tuerto o ciego' (kaszinahi)

'ruana' ([A] catuguai)

'espejo' ([A] canjoró; kanquexo)

'rayo', 'trueno' ([A] canchiratue; canchihi; [A] canchi)

'lo mató el rayo' (canchihi raquahi)

'boqueroncito' (canchiveja)

'canela ' ([A] cancrejó)

'espejo' (kanquexo; [A] canjoró )

'derribar' (kanszo-zá 'derriba')

'piña ' ([A] candejochi; cantejoche; cantijuchi)

'espina' ([A] candegache)

'coger' (canquehá? ‘ ¿cogieron?')

'cabellos' (quiexa)

'lejos' (quejará, quixarani 'vamos lejos')

'ser' (-qui, -qui-, quihi, quehi, quehé 'eres')

'venir' (quihi, qui, -que- quihi 'vine; quijra

'que venga'ba-que-zá 'ven')

'maíz' (kique, quique)

'no pariente' (quiqua quihi 'no eres mi pariente')

'trenzas' ([A] quinaja )

'cabeza' ([A] quinaji ; quinaxi)

'por la mañana' (quisicú )

'soplar' ( ko-zá 'sopla'; kaco? 'soplaste')201. kubiha

'puerta' (cobexa)

'caliente' (cubi-xa)

'anopta' ([A] cofi )

'estantillo' (cujuffi)

'casa o rancho' (cogo)

'cocina' (coguá)

'bagre, lechero ' [pez] ([A] cocoe)

'vender' (coma-zá 'vende')

'mayor' (cupihi) 
210. kuxuu

211. kukwa

212. kũbusa

213. kũki

214. kwa-

215. -kwa-

216. kwa-hurgihi

217. kwa

218. -kwa

219. -kwa

220. kwarihi

221. kwarií

222. kwaha-

223. kwahaka

224. kwahi-

225. kwahiru

226. kwahihi

227. kwahikihi-

228. kwahisu

229. kwahurukwa

230. kwahutga-

231. kwat ãkwa

232. kwat i-kwata-

233. kwatsihi-

234. kwatjuara-si

235. kwaja-

236. kwaja237. kwajuhu 238. kwakarai 239. kwakari-ari 240. kwakahu 241. kwakai 242. kwakakwa243. kwaku\$ti- 'casa' ([A] cojoo)

'cocina' (coguá)

'cerca' (cumbuzá)

'de brazos largos' ([A] conque )

'bueno' (nan-qua-há 'bueno está; [A] na-gua 'bueno')

'matar ' (canchihi raquahi 'lo mató el rayo';

niquaquani ' $i$..mata?')

'canasto' ([A] ochiji; guajuchixi 'canasta, canasto')

'cocinar' (-coa-,-qua- coa-gua 'cocina; naqua

niu-qua-ni '¿quién cocina?'

'hacer' (qua-ni-qua-jea 'hazme'; bi-qua-zá 'haced')

'decir' (ka-qua-ni 'i...dices?; -guabi 'digo' )

'codo' ([A] guariji )

'coger' ([A] fiera-cuare-jia 'coger'; ca-quanehé?

'¿cogiste?')

'subir' (guajazá 'sube')

'acordarse' (quajaga 'acuérdate')

'ir' (ninga buxi-bi 'yo me voy'; boxea-ba 'vámonos;

quaxe-ba, quexihá, cohagea 'anda; quaxihá ricá 've tú, [anda vos]')

'bambú' ([A] guajero )

'olla, cántaro' (guajixi, guafixi 'olla'; [A] guajije

'olla de barro redonda; [A] guajiji; 'olla de barro redonda peq.'); guaxixi 'cántaro')

'levantarse' (quafixijea 'levántate', fiquijizá 'levántate')

'guaduas' [bambú] (uhajizó; nuhaxi 'guaduales')

'despertar' (quajurogua 'despiértame')

'brincar' (huaxuchazá 'brinca')

'rayo' ([A] guachangua)

'topar' (ca-guachi-ni? '¿qué topaste?; ragua cha-nca

'nada he topado')

'entrar' (guachixizá, guachijiza 'entra')

'amarillo' (guachuara-zi)

'vivir, habitar' (coaya, caya- nincaxa coaya

'vive conmigo'; ningaxá caya-sesza '¿...vivir conmigo?’)

'sentarse' (coaya-za, coayá, caya-zá 'siéntate')

'lanza' (guayoxo; [A] guayojo)

'vaca' (guacaré, guacarahé)

'carne de vaca' (guacareare)

'canoa' ([A] guacajo; guaca ju[n]; guacaxu 'barqueta')

'sapo' (guacahi)

'caer ' (guaca qua-zá 'cáete!')

'abrazar' (guacundi-zá 'abrázame') 
244. kwakwa-

245. kwakwihahi

246. kwakwanaka

247. kwanisi-

248. kwaniahi-

249. kwanisi-

250. kwarusukwa

251. kwasa-

252. kwasi

253. kwasiãtisi

254. kwasiha-

255. kwasihi-

256. kwasimi-

257. kwasu

258. kwatihu nianu trinihu

259. kwatii kwatii-si

260. kwatii

261. kwaa-

262. kwahu

263. kwãbukai

264. kwãkwaa

265. kwãsưti

266. kwiku

267. kwiruniahu ‘algodón' (guahuahi; [A] guaguana )

'fibra, hilo' ([A] guaguejafi)

'muela' (juajuanaca)

‘borracho' (guanin szigaguehé? ‘ ¿estás borracho?’)

'lavar' (quanea jehá 'lava')

'reír' (huanisiza 'ríete')

'muchacha' (huaruzucoa)

'bautizar' (guazaba nincaxa 'te pondré nombre')

'mucho' (quazi)

'harto' (quasiantizi, joantizi 'harta')

'dejar ' (guasze fasza rincaxa 'déjamelo donde estoy')

'pasar' (guazixi-za , guasiji-zá 'pasa')

'hurtar' (huaszimî-zá 'hurta')

'huevo' ([A] guaso; huasho 'huevo de gallina')

'piedra de amolar ' (huatejo neanu chinexo)

'mate' (guatihi, batihi; (guatizi) 'matecito'

'piedra' (guatihi; [A] guatiye)

'dar' ([A] finguá 'yo te doy')

'ajî' ( guaxú ; [A] guajo ‘chile rojo enano')

‘cedro' (guambucahi)

'pelear' (-guancahá, quaquahá)

'barro; arcilla' ([A] guansuche)

'uno ', 'juntos ‘ (guhigo, gunfigo)

‘cuero' (cueronehaxo)

\section{M}

268. mabiha

269. mahanai

270. mahaa

271. mahihi

272. mahii

273. maĩtihu

274. matyii

275. matsitsi

276. manajai

277. manikwaka

278. manisa

279. masautsakwai

280. masĩkwa

281. masí

282. masuhuu 'culo' (mabexa)

'camarana' ([A] majanae)

'madre, mamá' (majahá; [A] maja )

'comida' (magixi)

'guacamayo' (majihi)

'coco' ([A] maindejo)

'sabaleta' (machihi)

'excremento' (machichi)

'lora' ([A] manayae)

'dardo' (maniguaca)

'bayeta' [tela de lana] (maneszá)

'ardilla' ([A] masauchaguai )

'rabo', cola' (maszengua; [A] maesegua )

'chonta' [palmera]; (maffi 'chontaduro')

'hoja' (masojo) 
283. masu

284. mãtinai

285. mãtisi

286. mãtukasu

287. mãtuna

288. mãne

289. mãsisai

290. mãtikwai

291. mãtinahu

292. mẽkwa-

293. mihi

294. mihina

295. mihinai

296. mihinai

297. mitrikia

298. mijã-

299. mikahi

300. -mikwa-

301. mikwai

302. mimi

303. misii

304. mitahu

305. mitai

306. mirfaru

307. mîku

308. mĩkunahui

309. mîkusa kwasa

310. mîkusua

311. mísara

312. mísara

313. mísikwahu

314. mísisai

315. míti-

316. muhanai

317. muhuasa 'plátano maduro' (mashú)

'cerdo salvaje' ([A] mandenae)

'sal' ([A] mandisi)

'plátano' ([A] mandugaso)

'nalgas' ([A] mandona )

'sardina' ([A] manne )

'tórtola grande', 'tórtola pequeña' ([A] mansesai, mensesai)

'murciélago' ([A] mandeguae)

'sachamate' (mantinaxo)

'besar' (menqua-zá 'besa')

'aguja' (mifi)

'tierra' ([A] mijina; jixena [ricaná], i...[tu] tierra?)

'perro' (mixinehi; mijinahé 'tu perro')

'tigre' (mixinehi shotajihi [shotajihi 'monte'];

[A] mijinae)

'picudo pequeño' ([A] michiguia)

'pedir' (miña-zá 'pide')

'maíz tostado' (micaffi)

'morir' (naqua nimiquani ‘¿quién muere?')

'tigre pequeño' ([A] miguae)

‘querer' (-miné, -mimi, mimi; ¿ca-miné? ‘ ¿quieres?';

ca-mimi '¿queréis?; quazi mimi 'mucho os quiero')

'hombre' (miszihi)

'cielo' ([A] mitajo)

'luna' ([A] mitae)

'flecha de bodoguera' (mincharo)

'vomita' (mingo-zá)

'pájaro' (mincunajuhi)

'blusa' ([A] mingocaguasa)

'brazo, brazos' ([A] mingosoa; mingoca)

'arena' (minzará)

'jagua ‘ [árbol] (minzará)

'arma', 'cuchara' (minsziguaxo, minchinaxo)

'tórtola pequeña' ([A] mensesai)

'estirar' (minti-zá 'estira')

'puerco saíno' (muxanahé)

'caraná' (mujuhaza) 
318. naa-

319. naatri

320. nahãsiţi

321. nahĩhusi

322. nahubaa

323. nakusiha

324. nakwa

325. namakwamana

326. nasui

327. nãhii

328. nãkii- rãkii-

329. nãkisi

330. nãkwa-

331. nãkwi

332. nãpakwana

333. nãsiisi anikwi

334. nãsiisi

335. nãtiniru

336. niaja-

337. nity $\mathrm{i}$

338. nikwaasi

339. nisĩhii

340. nĩkii

341. nîkwa-

342. nítii

343. nkakii

344. nsaasa

345. nsasi-

346. nsasi

347. nuja-

348. nusii 'llorar' (-na-, naha-; ni-na-quá 'no llores'; naha-zá 'llora')

'tarde' (nahachi 'que es tarde', 'ya es tarde'; raronahé 'ya es tarde')

'rabioso' (najanszichi ningaxa 'yo estoy rabioso')

'chonta' (naxinjuffi )

'lavar ' (nâjubahá 'a lavarnos')

'mañana' (nagosexa 'mañana; jana-gozexa 'iré mañana')

'quién' (naqua, naquanei, naquini, nagua, nuaqua)

'cera blanca' ([A] namauamana)

'romadizo' (naszuhé)

'cangrejo' (nanjihi)

'traer' (nanqueha, -nanquea, nangue, nanque, -ranquihi, naquiqua-nanqueha,gua-nanquea 'trae'; cane-nangue, nanque-za 'trae'; na-ranquihi '...me trajo'; naqui-guani? '¿...te trajo?')

'carne' ([A] nanquise; nanguize; nanquizi, naûquizi[n]) 'bonito' (nanqua-zi 'bonito'; nanquagaqui 'eres bonita') 'pava' (nangohé)

'veneno' ([A] nampaguana)

'tres' ( nanszihisze haniguhé )[dos + uno]

'dos' (nanszihiszi)

'totuma' [calabaza] ([A] nandinero)

'vivir, habitar ' (nehayaya, neayayá 'viven')

'cera de colmena' (nichi)

'calentura' (niquahaze)

'llover' (nizinxé 'ya llueve'; yazi misinjehé

'mucho llueve')

'pescado' (nenguihi, nêguihi, nengui, nengihi )

'aprender' ( ninqua-zá '‘aprende!'; ninquaraza 'aprende')

'ahogarse' (nintihi 'se ahogó')

'tener' (encaqué,ncaquehé,ncaque,kaque, kaquij,

'¿tenéis...?'ncá 'tengo')

'cuándo ' (aszâhaza, nszahaza, nszâ haza?)

'enfermo' (nszâzincahá 'estuve enfermo; nszarincaque?

‘¿estás enfurmo?')

'cómo, qué' (sazi, nszazini? ‘cómo', nszâzi ‘qué')

'temer ' (nuya chaé 'tengo miedo; nuya coá

'no tengas miedo')

'piojos' (nozihi) 
349. nusũkwa

350. nũki-

351. nũsuihu

352. nũsui

353. paka

354. pakaraĩkwa

355. pakaa

356. pakwaty $\mathrm{i}$

357. pakwaty ii

358. para

359. para

360. pasikai

361. patuhuu

362. pii

363. pikãfi

364. pikãtu

365. pisii

366. pitukwai

367. pihu

368. pi

369. pitikwana 'mujer' (nozuqua, chinizumqua[n]; huaruzucoa

'muchacha')

'mirar, ver' (nunquizá 'mira, ve')

'peje' (nunszuejo)

‘boquichico' (nuszuhé ; [A] musoe; 'bocachico'; noszue, 'pescado'; nunszuejo 'peje' )

\section{$\mathbf{P}$}

'yuca' (pagá; [A] pagà )

'parida' (pagaraengua)

'no tengo, no hay' (pajaá, pagá, pacahá, paxa, pagahá, pajá,paga, pajahá)

'calabaza' ([A] paguachí)

'zapallo' (paguachihi)

'nada' (para) [pará riszifigua 'no quiero beber más']

'no' (pará)

'gallinazo' (paszecahi))

'hoja' ([A] patojuhú)

'hermano' ([A] pii )

'banco' (picanffi)

'mesa' (picanffi )

'gallo' (piszihi)

'rana' (pituguahé)

'barbacoa' (pexó)

'andar' (campin cague ‘ ¿sabéis andar?’)

'patio de casa' (pintiguana)
370. -ra

371. rahãkaa

372. rahatats $\mathrm{i}$

373. rahi-

374. rahiabihisi-

375. rahikwahu

376. rahinatsii- 'locativo' ( cogora 'a casa'; zotajera 'al monte'; jicora 'a la iglesia')

'pobre' (ringa rajancahá 'yo soy pobre')

'amanecer' (rajatachi 'ya amanece')

'llevar' (raji-, raxi-, -ragea, -reajearajiza , raxizá 'lleva'; choragea 'quiero llevar'; quareajea 'llévame')

'nadar' (rajibijizin-, rajeari jizin-, cajaibi jin-)

'brazo de río' (rafeguaxo)

'anochecer' (raxinachihic 'anocheció';caxi nachihi

'ya anocheció',raxi nachichi 'ya es de noche') 
377. ramai

378. rasũkwai

379. rãkai

380. rãpaki

381. riarítii

382. riãsa-

383. riãtihi

384. rihiakai

385. rihũti-

386. rii-ni

387. rii-si

388. rika

389. rikii

390. rikuhu

391. rikwãtu-

392. rinikurãbia

393. risĩkaa

394. risi-

395. risihahi

396. rĩbii

397. ríka

398. ruhuii

399. rumakai

400. rumina

401. rũpakwasa 'morir ' (ramahi 'murió; ramaficora 'no muráis')

'tonto' (rashunguahé)

'podrido' (rancahi)

'bagre ' [pez] (rampaqui)

'tronar' (canchihi rearintihi 'truena') [lleva 'trueno'

como posible sujeto]

'dejar' (rahasza ficoha 'no quiero dejarte'; ficaca neasza-cohá 'no me dejes solo')

'calabaza' ([A] mandinifi; nandinero 'totuma';reantiffi' calabazo')

'honda' ([A] difiacai)

'fajarse' (rijunte-zá ‘fájate')

'aquí' (rini)

'este' (rihizi)

'tú , vos' (ricá, rica)

'mama' [diminutivo] ([A] riquii )

'bodoquera' (ricoxo)

'creer' (rihuantozá 'cree' ; riguantoza 'creed')

'rastro o huella' (rinicurambia)

'sí' (rizincaha)

'beber' (riszi-, resze-; riszi-zá ‘bebe'; ca-resze-recá

'¿ya bebiste?')

'bebida' (riszijaxi)

'tórtola' (rimbihi)

'yo' (ninga, ringa, dinga; rincaxahá 'a mí')

'frío, tener' (ruxuhihi 'tengo frío')

'pedernal' (rumagay)

'juansoca' ([A] rumenà)

'veneno' (rumpaguaza)

402. -sa

403. sahanii

404. sahaa

405. sahii

406. sahunu-

407. sahu

'formador de imperativo' -za ( kaje-zá 'lava'; chuhua-zá 'oíd'; chiya-zá 'come!'; fifi-zá 'llama')

‘dónde' (szajani, szafanihi, szajamnszâjini ¿dónde está?; ¿sxaxini ‘dónde están? ; ¿szafanihi? 'dónde’;

caxini 'cuál' nsza jini'cuál'; ¿szajaconi? 'por dónde')

'quedarse' (zajahá 'me quedo')

'laguna' (szajihi)

'echarse '(fsrrajonozá 'échate')

'chapul’ [libélula] (szajohé) 
408. saikã-

409. satri

410. sakãţii

411. saka

412. sakakwaa

413. sakanahi

414. sakanahu

415. sakanihi

416. sakaa

417. saku-

418. sakwã kwał̧uakwa

419. sakwa

420. sakwai

421. samunii

422. sana

423. sanai

424. sanaku

425. sani

426. sani

427. sanihanua

428. sanika-

429. sanuhi

430. sapajahu

431. sasakwana

432. sasi

433. sasihakwa

434. sasihai

435. sahu

436. sai

437. saka

438. sasa

439. sãbina

440. sãhikaka

441. sãhinui

442. sãkaa-

443. sãkwa

444. sãkwaka

445. sãkwaa-

446. sãtini 'miel de caña' (szahicanxe; szahicaha 'caña dulce')

'aguacate' ([A] sachi; szachi)

'gavilán' (saganchihi)

'bejuco, caña' ([A] sacca)

'tropezar ' (nifsrraca quahá 'no tropieces'; fsruaquahá 'tropecé')

'rodilla' ([A] sacanaji)

'rodilla' (szacanaxo)

'estaca con horqueta' ([A] sacanifi)

'mano, manos' ([A] sacaà; sacahá)

'escupir' (fsrracozá 'escupe')

'cinco' (saguan cuachoagua)

'bejuco guamo' (fsrragua)

'cejas' (szaguahe)

'garrapata' (szamunihi)

'dedo' (sana)

'sardina' (szanahi)

'sardina' ([A] sanagó)

'esperar' (sani 'espera')

'ahora' (sáni)

'enseñar' (fsrranijanôhá 'te enseñaré')

'tener, sostener, coger' (sanica-zá 'ten')

'saliva' (fsranoxe)

'papaya' (sapayaxo; [A] sapallajò)

'barbilla' [hueso] ([A] sasaguana)

'dedo' ([A] safsri)

'prima' (szasejagua)

'primo' (szasejahé)

'uñas' (saxó)

gusano' (szahé)

'rige o exonera' (szagá)

'ropa, enagua, camiseta' (saszá )

'empeine' (sambenà)

'agave americana' [pita] ([A] sanjecacà )

'alacrán' (szanjenohé)

'hablar' (zanca-zá 'habla',zancanca juatuszá

'hablo derecho';zancahaya 'están hablando';

quanazancahá nâ yaqui hizihi 'cuéntame tus trabajos')

'cuñada' (zangua)

'cuello' ([A] sanguaca )

'dar' (ni-fsrranqua-za, quanin-fsrruangua, quane-ffranquane, 'dame'; ni-fssrraquahá 'daréis')

'despacio' (santini) 
447. sira

448. - si

449. siãbina

450. siãbunaka

451. siãkwahuri

452. siãkwaka

453. siahi-

454. siakwaja

455. siha

456. sihajaha

457. sihana

458. sihi

459. sihiihu

460. sihuhi

461. sijasa

462. siju-

463. sijuhu

464. sijui

465. sika

466. sikaa

467. siku-

468. siku-

469. sikwihu

470. simiahasi

471. sinasa

472. sinihi

473. sipu-

474. sisa

475. sisuhi

476. sitahi

477. sitii

478. síkinai

479. sísihi

480. sísihui

481. siũ kai

482. skãhi akũ kwabi

483. suata

484. suãsa-

485. sukara

486. surunia ‘ceniza' (szira)

'formativo de adjetivos'; (guachuara-zi ‘amarillo'; aiszimi-zi 'azul' ; finszi-zi 'sucio')

'peine' (zeambina)

'cumbrera' (zeanbunaca)

'cerebro' (szean quafori)

'pescuezo, cuello' (szeanquaca; [A] sanguaca)

'olvidar ' (-fsrraji-, -fsrriaxi ; nifsrrajigua 'no te olvides' cafsrriaxi? '¿te olvidaste?)

'mono volador','mono coli-largo'; ([A] seguaya;

[A] fiagua)

'cabuya' (fsrrixa)

'alma' (szejayaxá)

‘pecho' (szixaná)

'ojos' ([A] sifi; szifi)

'cejas' ([A] sifiijo)

'sombrero' ([A] sijofi)

'estar' (ziyaza 'estaremos')

'moler' (siyozá, ziyozá 'muele')

'tortilla' (ziyoxu)

'mono' (ziyuhé; sziyuhyé 'mono chorro')

'orines' (zega)

'pie o pierna' (szecahá)

'dientes [con] grandes' ([A] sicoji)

'diente, dientes' (szicoca; [A] sicoga)

'zapatos' (szicuhixo)

'chaquiras' (szimeafazi)

'pestaña' (szinasza)

'enaguas' (siniji)

'sopla' (zipo-zá)

'nombre' (sisza)

'leche' (szeszoxé)

‘cerro' (szitafi)

'chucha' [zarigüeya] (szitihi)

'mico chiquito' (zinquinahi)

'corazón' ([A] sinsije)

'pariente' ([A] sensejoe)

'guara, guaratinajo' ([A] siñocae; sziñucahé)

'un poquito' (szcanje agum guabi)

'callar' (shoata, fsâtani 'calla')

'levantarse' (soanza coaya 'no te levantes')

'oro' ([A] socara )

'perdiz grande' ([A] soronea) 
487. suhii

488. suhubi

489. sui

490. sujãsa

491. sukahi

492. sukahu

493. sukai

494. sukurusi

495. sukwi

496. sukwapana

497. sumukai

498. sunai

499. sunasu

500. susi-

501. susitg $i$

502. susuka

503. suta-

504. sutahii

505. sutakwa

506. sutusa

507. sũhuu

508. sũţua

509. sũkwahi

510. sũkwa-

511. sũkwahu

512. sũkwai

513. sũkwaraa

514. sũtai

515. sũti-

516. sũtihu

517. sũtihui

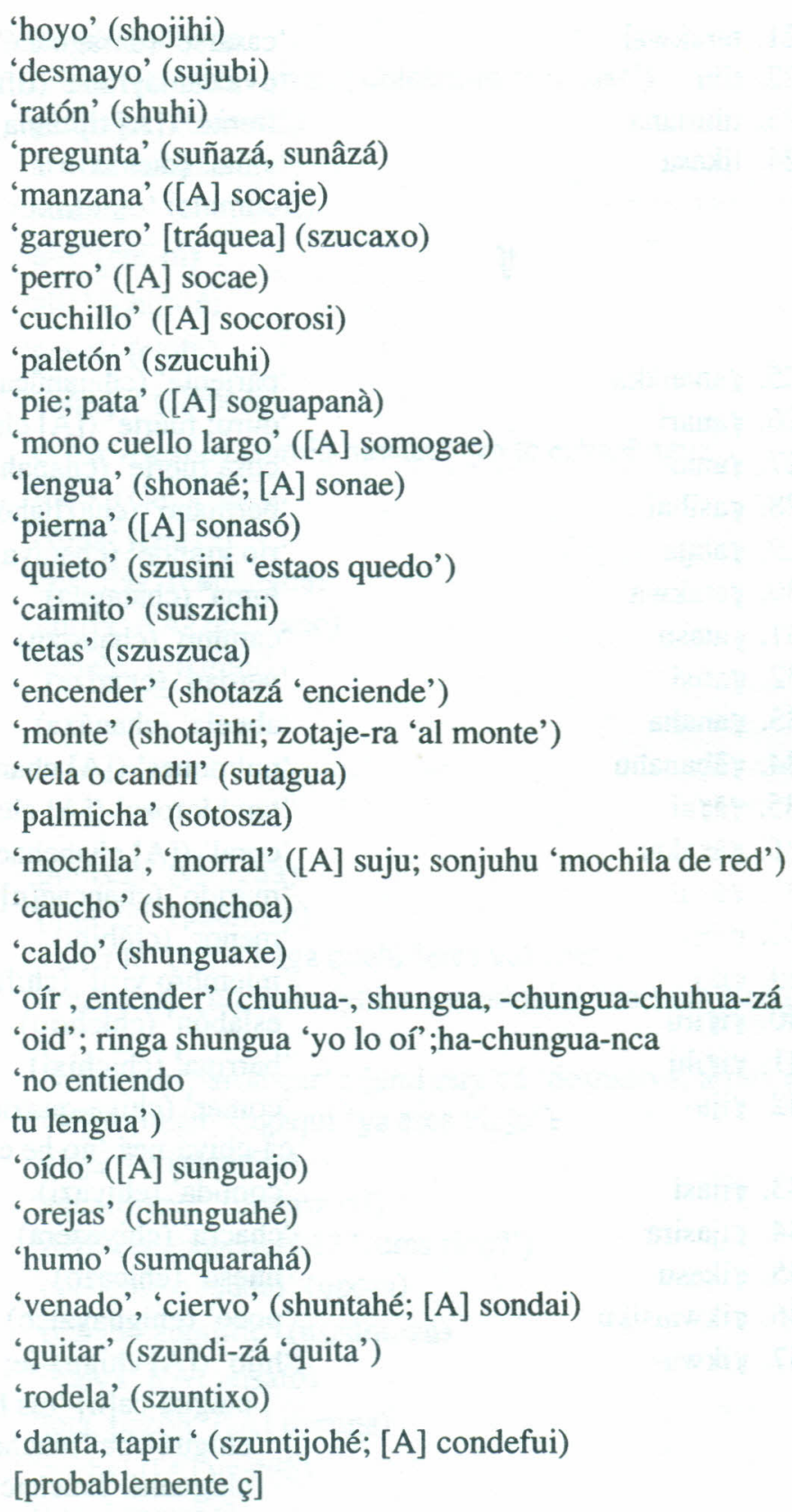


521. tarakwai

522. tihi

523. tihitiana

524. tikasa

525. ๖ahanũka

526. tranari

527. tsanai

528. trasihai

529. trataja

530. tatakwa

531. tratasu

532. tratisi

533. ty anaha

534. tsãbanahu

535. țãţai

536. trãtsakai

537. t ãkai

538. ษ ibii

539. tsihi

540. ty itgiru

541. ts itsihi

542. tija-

543. trijasi

544. t $\mathrm{ijasira}$

545. tikasu

546. tikwaisiku

547. tikwa-

548. tikwaka

549. trikwai kwakara

550. timanasu

551. t inarai

552. trinasa

553. ts inats ini

554. tsiniku ‘casarse' (daraguahé? ‘quiere casarse?’)

'uva camayrona' (tiffi)

'frente' ([A] tijitiana)

'cinta' (dicazá)

'pariente' (chajanûca quihi 'eres mi pariente')

'duro; fuerte' ([A] chanari)

'cosa fuerte' (chanahi )

'hermano' (chazijahé, chazajahi)

'río grande' (chataya)

'loma' (chatagua)

‘camino' (chataszu)

'verdad' (chatize)

‘abuela' (chanáxa)

'pulmones' ([A] chambanajo)

'tembloroso' ([A] chanchae)

'coral' ([A] chanchacae)

'marido' (chancae[n])

'menor' (chibihi)

'miembro viril' (chifi)

'eslabón' (chichiro)

'barriga' (chichixi)

'comer' (chiya-quazira chiya-ya 'todo han comido' ca-chiya-ncá 'no he comido'; chiya-zá 'come')

'comida' (chiyazi)

'chacra' (chiyazera)

'hueso' (chicazo)

'poco' (chiguayzico)

'hijo' ([A] chigua-ie; chigua-ca 'chiquillo'; chigua-ca; chigua-ca[n] 'tus hijos'; [A] chigua-gus 'nieto';

chigua-coa 'muchacha'; [A] chiguaco 'muchacho'; chiguahé 'muchacho'; [A] chiguagua 'primo')

'pecho' ([A] chiguaga)

'becerrito' (chiguahé guacarahé)

'plátano guineo' ([A] chimanazo; chimanazo)

'roca' ([A] chinarae)

'labios, boca' (chinaszá)

'al otro lado' (chinachini)

'por allî' (chinicú) 
555. ripina

556. riisi-

557. risuhu

558. ชitaryi-ni

559. ríbuhi

560. ชîi\$-ni

561. rii \$-ta

562. ชii

563. ชíkai

564. ชsũmia

565. ชũkaa

566. ruha

567. ruhukwa

568. rukumi

569. rupii

570. urihikwa

571. ubanuku

572. uranaka

573. umanii

no'

574. umikwi-

575. unãkaka

576. unãkakwa

577. unãsahi

578. unahaka

579. upura

580. usũtusu

581. uีsahu

582. ũsuka

583. ũsuhu

584. uีtai

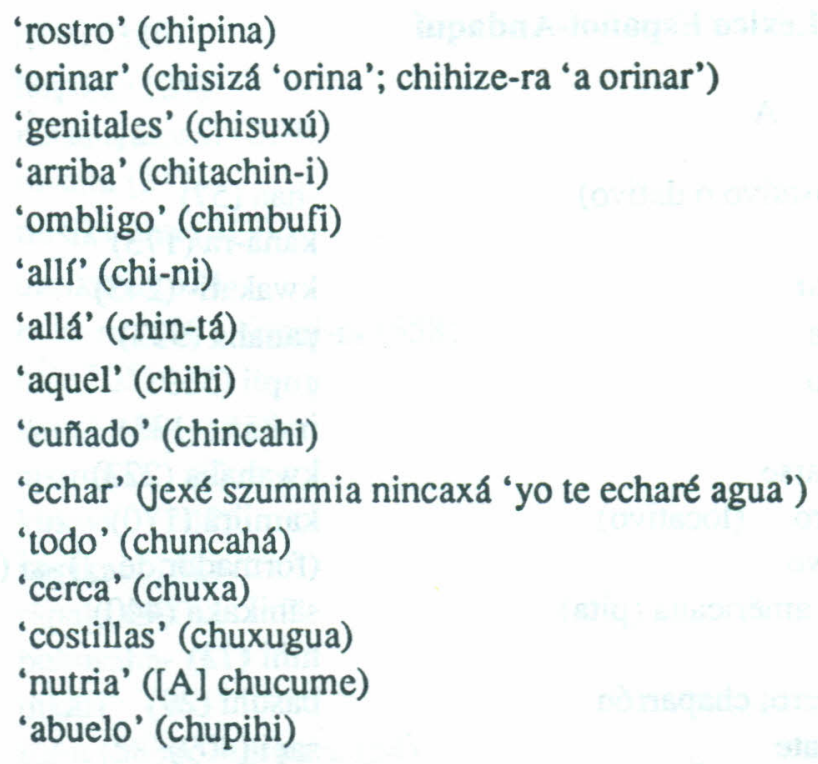

'preñada' (vrijigua)

'plumaje' (ubanôco)

'valiente' (vcha naga quehi 'eres valiente')

'mezquino' (umanihi, yhiza vmaniquini 'no seas mezqui-

'desherbar','arrancar' ; (umi cuy-zá 'deshierva, arranca')

'viejo' (unam kagaqui 'ya eres viejo')

'vieja' (unancagua)

'aguardiente' (unanszaxi)

'rico' (unajaga quehé? ‘¿eres rico?')

'corriente o raudal' (upora)

'plátano amarillo' (uszûntuzu)

'malva' ([A] unsafo)

'teta, pezón' ([A] unsuga)

'barba' ([A] unsojo)

'luna' (untahé) 


\section{Léxico Español-Andaquí}

A

a (acusativo o dativo)

-haa (57)

abajo

kana-ra (173)

abrazar

kwakưti- (243)

abuela

tyanaha (533)

abuelo

tsupii (569)

acero

iraharu (133)

acordarse

adentro (locativo)

kwahaka (223)

adjetivo

agave americana (pita)

kamiira (170)

(formador de _ ) -si (448)

sãhikaka (440)

agua

hihi (73)

aguacero; chaparrón

basuhi (29)

aguacate

satyi (409)

aguardiente

unãsahi (577)

aguja

mihi (293)

ahogarse

nitii (342)

ahora

sani (426)

ají, chile rojo enano

kwahu (262)

alacrán

alegre

sãhinui (441)

kamini (171)

algodón

kwakwa- (244)

al otro lado

trinats ini (553)

aquel

ขร $\overparen{\mathrm{ii}}(562)$

allá

t)

allí

tร ii-ni(560)

alma

sihajaha (456)

alzar

ãta- (13)

amanecer

rahatats $i$ (372)

amar, querer

hiãsu- (71)

amarillo

amarrar

amolar

kwatfuarasi

hiríhi (70)

kanu- (176)

andar

-pí (368)

anochecer

rahinatsii- (376)

anopta

kuhi (203)

anzuelo

hutsikwa (127)

aprender

níkwa- (341)

este

rii-si (387) 


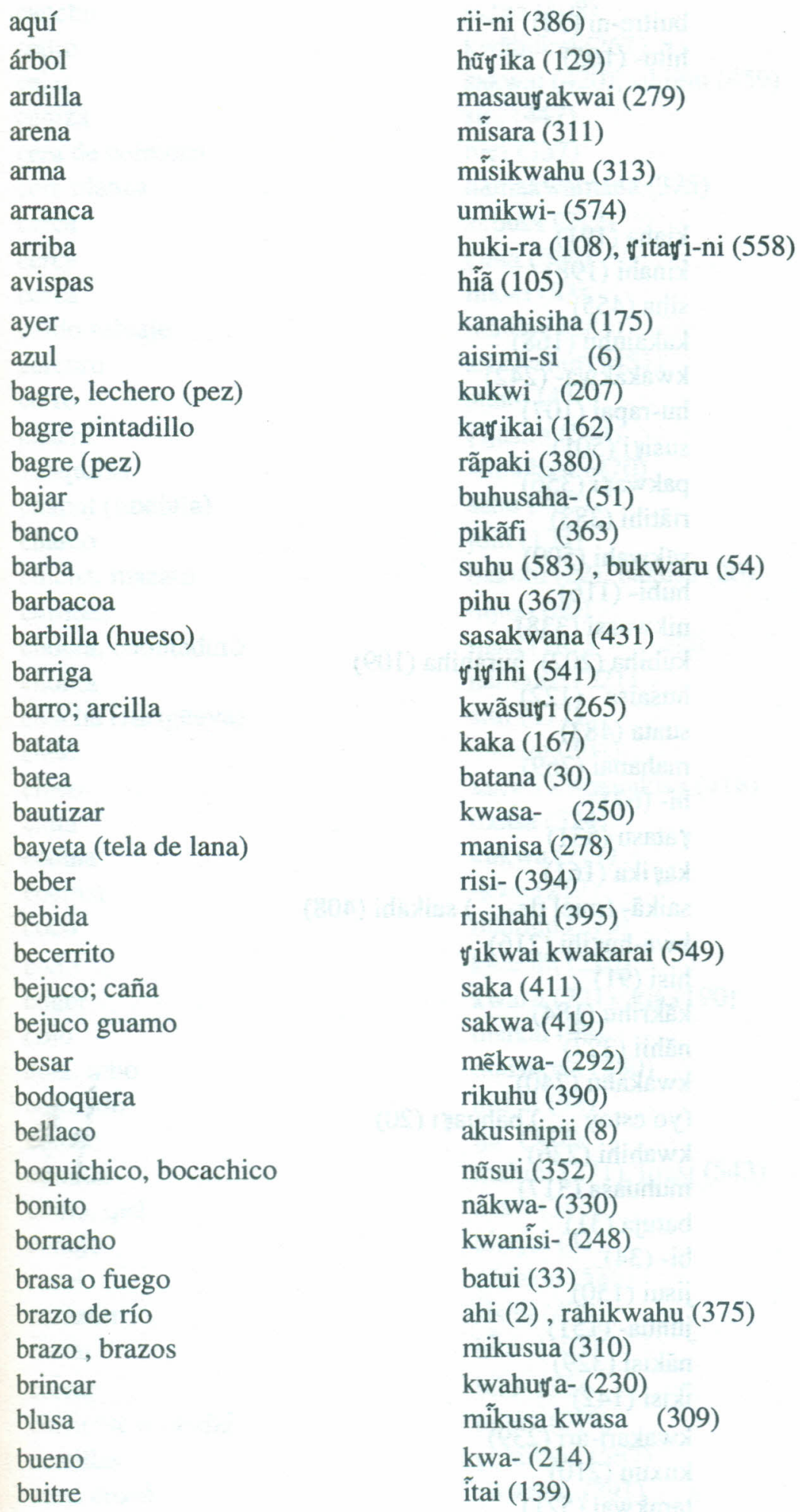


buitre

buscar

C buitre-ni (52)

hitu- (105)

kiaha (191)

kinahi (198)

siha (455)

kakaiuhu (168)

kwakakwa- (242)

hu-rapai (107)

susitri (501)

pakwatgi (356)

riãtihi (383)

sũkwahi (509)

hubi- (116)

nikwaasi (338)

kubiha (202), hurahiha (109)

husaisa (122)

suata (483)

mahanai (269)

hi- (67)

tratasu (531)

katfika (161)

saikã- (miel de _ ) saikãhi (408)

kwa-hutrihi (216)

hisi (91)

kãkrihu (184)

nãhii (327)

kwakahu (240)

(yo estoy _ ) bahuatsi (20)

kwahihi (226)

muhuasa (317)

batuja (31)

bi- (34)

jisui (150)

juhua- (151)

nãkisi (329)

îkisi (142)

kwakari-ari (239)

kuxuu (210)

tarakwai (521) 


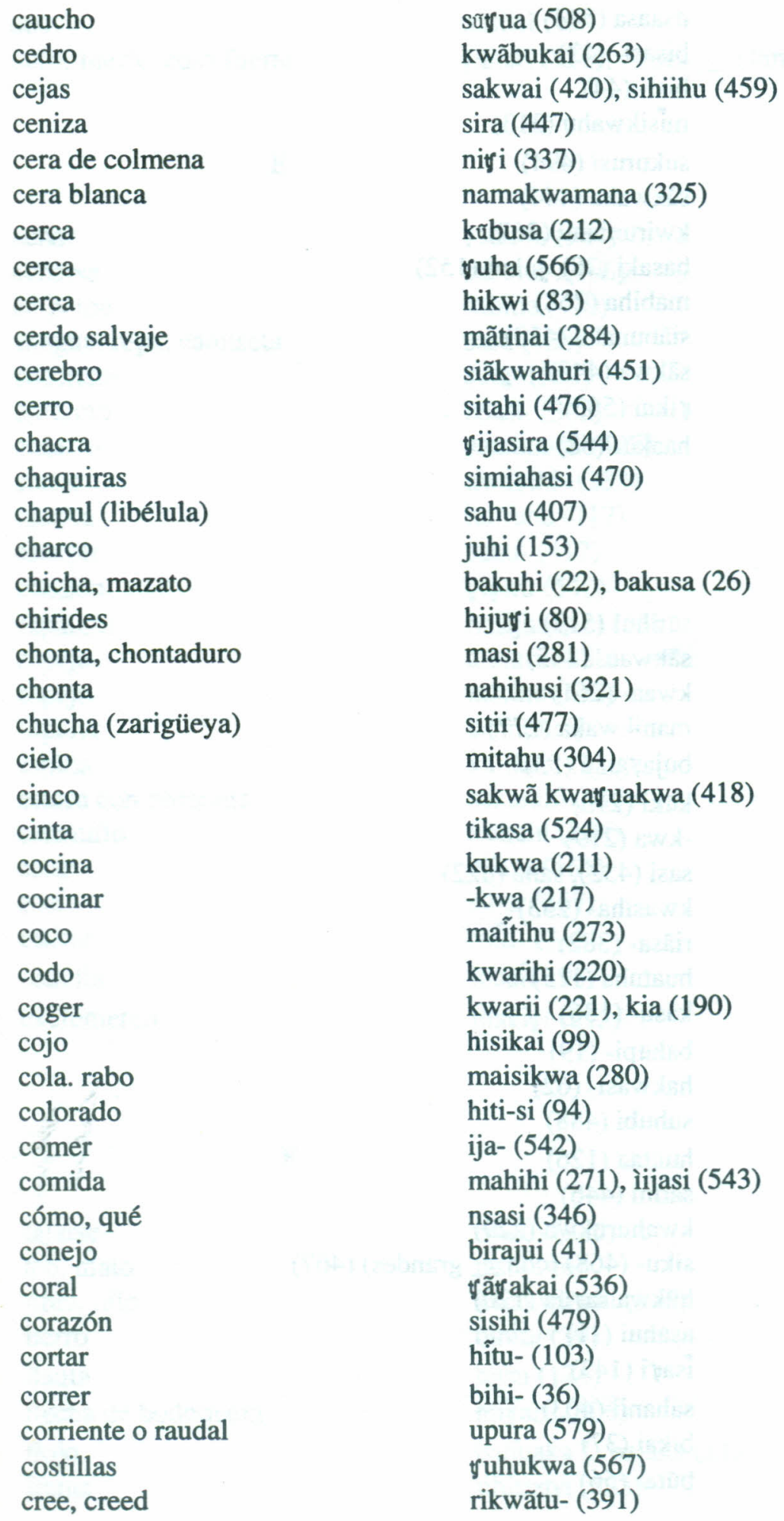




$\begin{array}{ll}\begin{array}{l}\text { cuándo } \\ \text { cuánto }\end{array} & \text { nsaasa (344) } \\ \text { cuántos } & \text { bisasi- (42) } \\ \text { bisi- }(44) \\ \text { cuchara } \\ \text { mísikwahu (313) } \\ \text { cuchillo } & \text { sukurusi (494) } \\ \text { cuello } & \text { sãkwaka (444) } \\ \text { cuero } & \text { kwiruniahu (267) } \\ \text { culebra } & \text { basaki }(28), \text { juhui (152) } \\ \text { culo } & \text { mabiha (268) } \\ \text { cumbrera } & \text { siãbunaka (450) } \\ \text { cuñada } & \text { sãkwa (443) } \\ \text { cuñado } & \text { tikai }(563) \\ \text { curar } & \text { haakũ- (58) }\end{array}$

D

\begin{tabular}{|c|c|}
\hline danta, tapir & sũtihui (517) \\
\hline dar & sãkwaa- (445) \\
\hline dar & kwaa- $(261)$ \\
\hline dardo & manikwaka (277) \\
\hline dátil & bujats anai (53) \\
\hline de brazos largos & kũki (213) \\
\hline decir & -kwa (219) \\
\hline dedo & sasi (432), sana (422) \\
\hline dejar & kwasiha- (253) \\
\hline dejar & riãsa- (382) \\
\hline derecho & huatuha (115) \\
\hline derribar & kãsu- (186) \\
\hline desatar & bahũpi- (19) \\
\hline descansar & hakwasi- (62) \\
\hline desmayo & suhubi (488) \\
\hline desnudo & huũtaa (126) \\
\hline despacio & sãtini (446) \\
\hline despertar & kwahurukwa (229) \\
\hline diente, dientes, & siku- (468) (con _ grandes) (467) \\
\hline diez días & hukwa satsua (120) \\
\hline Dios & asahui (11) \\
\hline doler & isatgi (143) \\
\hline ¿dónde? & sahanii (403) \\
\hline dorada & bikai (37) \\
\hline dormir & bũta- (56) \\
\hline
\end{tabular}


dos

duro; fuerte; cosa fucrte

E nãsiisi (334)

tanari (526), ( cosa _ ) ìanai (527)

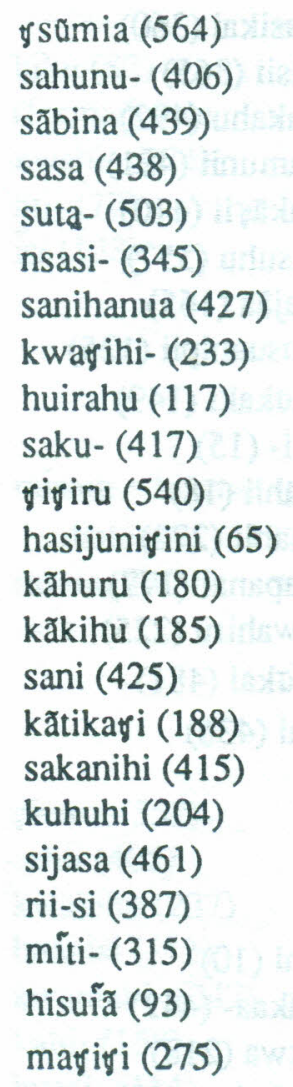

F rihũti- (385)

jasi (146)

kwakwihahi (245)

binítu (39)

husu (124)

mirraru (306)

bahuaka Ú bajaka (21)

tihitiana (523) 
frio

frio, tener

gallina

gallinazo

gallo

garguero (tráquea)

garrapata

gavilán

genitales

gente; rio

gordo

grande

gritar

guacamaya

guacamayo

guacamaya

bambú; guaduas; guaduales

guara, guaratinajo

gusano

G bianaha (35)

ruhuii (398)

harakwã̃ (64)

pasikai (360)

pisii (365)

sukahu (492)

samunii (421)

sakãryii (410)

ซisuhu (557)

kajãa (165)

husuanipii (125)

jãũaki (149)

ãti- (15)

bahii (18)

mahii (272)

kapanai (177)

kwahiru (225)

síũkai (481)

sai (436)

H

haber

hablar

hacer

hacha

hamaca

harto

harto

hermano

hermano

hígado

niño, hijo

hoja

hombre

hombro

honda

hoyo

ani (10)

sãkaa- (442)

-kwa (218)

buhukaa (50)

ihu (130)

kwasiãtisi (252)

hakưri (61)

pii (362)

rasihai (528)

hihinatu (74)

yikwa- (547)

patuhuu masuhuu (posiblemente diferencia dialectal) (361)

misii (303), ( soy _ ) bisiika (45)

huana-hi (112)

rihiakai (384)

uhii (487) 
hueso

huevo, huevo de gallina

hurtar

I

iglesia

imperativo

ir

ir

ir trikasu (545)

kwasu (256)

kwasimi- (255) hiku (82)

(formador de _ ) -sa (402)

kwahi- (224)

hii- (77)

îsi (138)

J

jagua (árbol)

juansoca

mísara hisaraa (posiblemente diferencia dialectal) (312)

rumina (400)

L

labios, boca

trinasa (552)

laguna

lanza

largo

lavar

lavar

lavar

lavar

leche

lengua

lejos

levantarse

levantarse

levantarse

limón

limpiar

llamar

llano, llanos

llanto

sahii (405)

kwajuhu (237)

huanisi (113)

kwaniahi- (247)

kahi- (158)

huasi- (114)

nahubaa (322)

sisuhi (475)

sunai (498)

kihara (192)

kwahikihi- (227)

suãsa- (484)

buhũsi- (49)

akai (7)

hîsu- (101)

hihii- (75)

hahana (59)

banukutfu (27)

rahi- (373) 
llorar

llover

locativo

loma

lora

loro, loros

luna

luna naa- (318)

nisĩhii (339)

-ra (370)

ţatakwa (530)

manajai (276)

kabii (157)

mitai (305)

ũtai (584)

M

macana
machete
madre, mamá
madre
maíz
maíz tostado
marido
tusa de maíz
mazamorra
malva
mama (diminutivo)
mañana
mano, manos
manzana
matar
mate grande
mate, matecito
mazato
mayor
medias o botas
mediodía
medir
menor
mentira
mesa
mezquino
miembro viril
mico chiquito
miel
miel de abejas

bitunahi (46)

binihi (40)

mahaa (270)

katfaa (160)

kiki (195)

mikahi (299)

t๘ãkai (537)

hitikai (102)

kaihi (159)

ũsahu (581)

rikii (389)

nakusiha (323)

sakaa (416)

sukahi (491)

-kwa- (215)

hãjũi (66)

kwatii, kwatii-si (259)

bakusa (26)

kupii (209)

akwajuhu (9)

isahura ii (134)

bitfi- (47)

tribii (538)

itfua (140)

pikãtu (364)

umanii (573)

trihi (539)

sikinai (478)

bakwi (23)

bakwihi (23) 


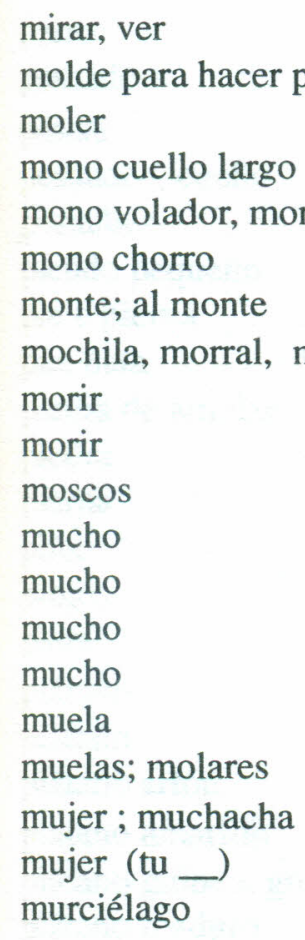

nũki- (350)

ikuhu (132)

siju- (462)

sumukai (497)

siakwaja (454)

sijui (464)

sutahii (504)

sũhuu 507)

ramai (377)

-mikwa- (300)

hupinai (121)

huãti (111)

jasimi (147)

bikwara (38)

kwasi (251)

kwakwanaka (246)

ahubinaka (4)

nusũkwa (349)

karupikwa (155)

bîtikwai mãtikwai (48)

para (358)

rahiabihisi- (374)

mãtuna (287)

îkihi (141)

hinai (87)

hisasa (90)

kaţ inikwa (164)

katy initgi (163)

trikwa- (547)

kamija (172)

para (359)

sisa (474)

kikwa (196)

pakaa (355)

tsukumi (568) 
O

oído
oír, entender
orejas
ojos
olvidar
olla; olla de barro redonda
ombligo
orinar
orines
oro
oro

sũkwahu (512)

sũkwa- (510)

sũkwai (513)

sihi (458)

siahi- (453)

kwahihi (226)

tríbuhi (559)

tfiisi- (556)

sika (465)

isikara (135)

sukara (485)

\section{$\mathbf{P}$}

padre; papá
pájaro
paletón
palmicha
papaya
¿para qué?, ¿para qué
parida
parir
pariente
pasar
patio de casa
paugi (ave gallinácea)
pauji
pava
pecho
pecho
pedernal
pedir
peine
pelear
perder
perdiz pequeña
perdiz
perdiz grande
pereza
periquito

tahaa (518)
míkunahui (308)
sukui (495)
sutusa (506)
sapajahu (430)
hisaha- (70)
pakaraîkwa (354)
-taa (519)
tahanuka (525)
kwasihi- (255)
pítikwana (369)
tai (520)
hitii (96)
nãkwi (331)
tikwaka (548)
sihana (457)
rumakai (399)
mijã- (298)
siãbina (449)
-kwãkwaa (264)
-hiusi (97)
bukwakai (55)
hurukwakai (110)
surunia (486)
ahuma- (5)
hijutribi (81)




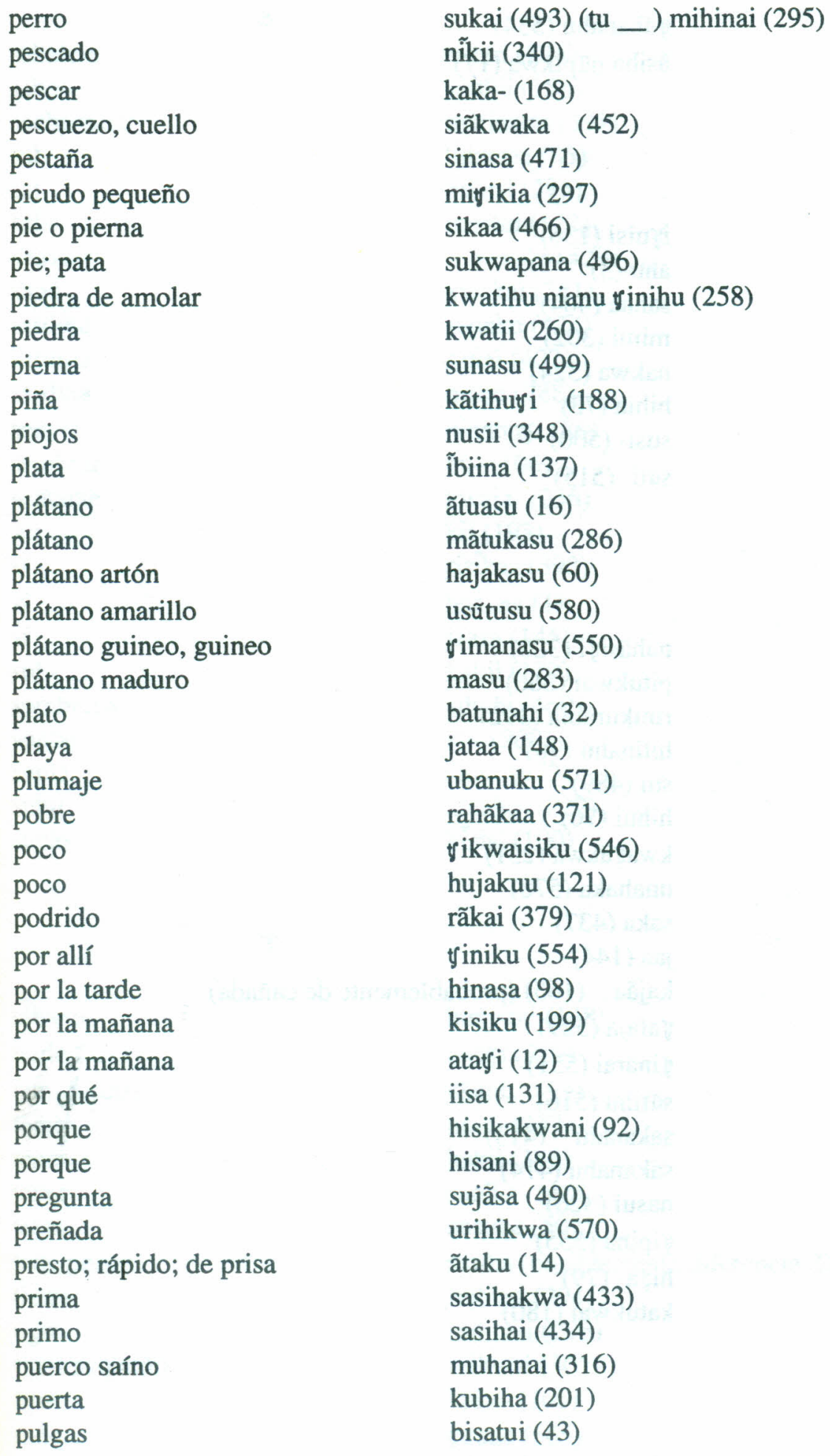


pulmones

puta o ramera

Q

qué

quebrada

quedarse

querer

quién

quien sabe

quieto

quitar

$\mathbf{R}$ tfãbanahu (534)

ãsiha nũpikwa (17) itfuisi (136)

ahu (3)

sahaa (404)

mimi (302)

nakwa (324)

hihia (73)

susi- (500)

sũti- (515)

nahãsiţi (320)

pitukwai (366)

rinikurãbia (392)

hitinahu (96)

sui (489)

hihui (76)

kwatãakwa (231)

unahaka (578)

saka (437)

jaa (144)

kajãa (165) (probablemente de cañada)

tyataja (529)

tyinarai (551)

sũtihu (516)

sakanahi (413)

sakanahu (414)

nasui (326)

tripina (555)

hitfa- (79)

katukwai (180) 
S

sabaleta

saber

sachamate

sal

sal

salir

saliva

sapo

sardina

sardina

sardina

sed

sembrar

sentarse

ser

sí

sí

sí

sol

sombrero

soplar

sopla

subir

sucio

tábano

tarde

tembloroso

temer

tener

tener

tetas

teta, pezón

tierra

tigre

tigre pequeño

tirar

todo matsii (274)

kaakii (156)

mãtinahu (291)

hikwasi (85)

mãtisi (285)

husihii (123)

sanuhi (429)

kwakai (241)

sanaku (424)

sanai (423)

mãne (288)

hinahutsi (86)

hu- (106)

kwaja- (236)

kii (193)

risî́kaa (393)

haa, aa (1)

jaasa (145)

kakii (170)

sihuhi (460)

ku- (200)

sipu- (473)

kwaha- (222)

hîsisi (100) hutfunai (128)

naatfi (319)

tyãţai (535)

nuja- (347)

kakii (343)

sanika- (428)

susuka (502)

ũsuka (posiblemente diferencia dialectal) (582)

mihina (294)

mihinai (296)

mikwai (301)

kaka- (166)

țũkaa (565) 
tonto

topar

torcido

tortilla

tórtola

tórtola

traer

trenzas

tres

tropezar

tronar

trueno; rayo

tú; vos

tuerto o ciego

$\mathbf{U}$

uno; juntos

un poquito

uñas

uva camayrona

V

vaca

valiente

vela o candil

venado, ciervo

vender

veneno

venir

venir

verdad

vieja

viejo

viento

vivir, habitar

vomita rasũkwai (378)

kwatsi- kwatsa- (232)

kanaha (175)

sijuhu (463)

( _ pequeña ) misisai (314),

( _ grande) mãsisai (289)

ríbii (396)

nãkii- rãkii (328)

kinaha (197)

nãsiisi anikwi (333)

sakakwaa (412)

riarítii (381)

kãtfi (182)

rika (388)

kasinai (179)

kwiku; kũhiku (266)

skãhi akũ kwabi (482)

sahu (435)

tihi (522)

kwakarai (238)

ut anaka (572)

sutakwa (505)

sũtai (514)

kuma- (208)

nãpakwana (332), rũpakwasa (401)

kii (194)

ju (154)

tyatisi (532)

unãkakwa (576)

unãkaka (575)

hikujãha (84)

niaja- (336), kwaja- (235)

míku (307) 


\section{$\mathbf{Y}$}

yo

rĩka (397)

yuca

paka (353)

Z

zapallo

pakwatyii (357)

zapatos

sikwihu (469)

\section{Bibliografía}

Albis, Manuel María. 1855. "The indians of Andaqui, New Granada" Notes of a traveller. Bulletin of the American Ethnological Society. Vol. 1 años 1860-1861.

Anónimo. 1928. Lenguas de América. Manuscritos de la Real Biblioteca. Vol. 1 (Catálogo de la Real Biblioteca Vol. VI). Madrid.

Constenla Umaña Adolfo. 1981 Comparative Chibchan Phonology. Tesis Doctoral. Universidad de Pensilvania.1992. El Método de Restitución. Comunicación personal.

Coronas Urzúa, Gabriela. 1994. "Análisis fonológico de la lengua andaquî". En Revista de Filología y Lingüística de la Universidad de Costa Rica. Vol. XX No. 1

Friede, Juan. 1953. Los Andakí (1538-1947). Historia de la aculturación de una tribu selvática. Fondo de Cultura Económica. México, D.F.

Haensch, Günther. 1982. La Lexicografía.. Madrid: Editorial Gredos.

Rivet, Paul. 1924. "La langue Andakî". En Journal de la Societé des Américanistes. París. Vol XVI. 


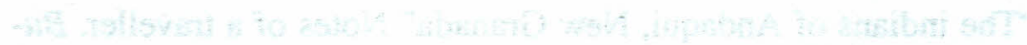

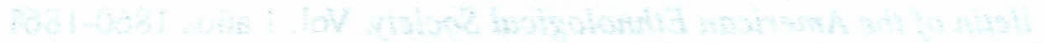

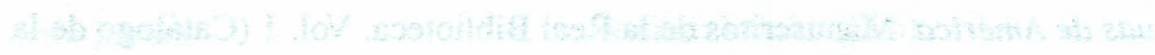

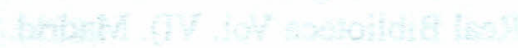

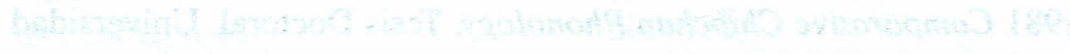

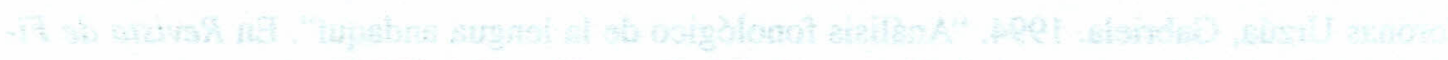

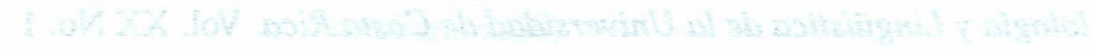

